\title{
Research on Structural Similarity Design Emergency Exercise's Scenario
}

\author{
Kaigong Zhao, Haiyan Wang ${ }^{(D}$, and Dengfeng Zheng \\ School of Civil and Resource Engineering, University of Science and Technology Beijing, Xueyuan Road, Haidian District, \\ Beijing 10083, China \\ Correspondence should be addressed to Haiyan Wang; whycumtb@163.com
}

Received 16 October 2021; Accepted 7 January 2022; Published 11 February 2022

Academic Editor: Peng Hou

Copyright $(92022$ Kaigong Zhao et al. This is an open access article distributed under the Creative Commons Attribution License, which permits unrestricted use, distribution, and reproduction in any medium, provided the original work is properly cited.

In order to better apply the "situational response" model in the field of fluidized mining emergency management, it is the first step and the most critical problem to construct a reasonable scenario for fluidized mining emergency drills and reasonably put forward emergency management measures. Therefore, the structural similarity method is adopted in this paper to design emergency exercise scenarios. Firstly, a model of hierarchical structured scenarios is proposed, namely, modules of "Event-EnvironmentState of scenario-Disposal of task-Emergency action- Resources subject." Secondly, a scenario chain is designed, and a prediction method of the event development trend under the current scenario is proposed. Thirdly, the calculation method of scenario similarity and the proposed emergency response scheme method under the current situation after similarity comparison are proposed. Finally, the structural similarity analysis method is used to verify the application of "scenario construction" in oil and gas pipeline accidents, and better analysis results are obtained. Through this research, the application of "scenario design" in fluidized mining emergency management has been expanded and enriched, and technical support for "scenario design" of fluidized mining assisted decisions is provided.

\section{Introduction}

The fluidized mining is a mining technology system that converts deep solid mineral resources into gaseous, liquid, or mixed gas-solid-liquid material in situ and achieves unmanned and intelligent downhole conversion of mining and charging, thermoelectricity, and so on. These transformed gaseous, liquid, or mixed gas-solid-liquid substances are flammable and explosive and are highly susceptible to combustion and explosive safety incidents [1]. In the event of such a safety incident, there would be serious implications for fluidized mining, and there is currently no relevant information available to provide a basis for emergency response measures in the event of an incident. Therefore, the emergency response capability preparedness for safety incidents during fluidized mining needs to be improved through emergency drill measures [2]. In recent years, the application of "scenario construction" technology in the field of emergency management has been given close attention by domestic and foreign academic circles, and its research results are undoubtedly one of the most cutting-edge issues in the field of global emergency management [3]. Emergency management often needs to improve targeted legal mechanism construction plans and their interoperability is not strong, and emergency team overall service quality has problems such as the allocation of resources. The real problem is that the traditional "predict-response" model is not very good at dealing with all kinds of emergencies. In today's society, "scenario-response" mode is needed. Therefore, it is an urgent need to apply "scenario construction" technology to the field of emergency management.

In the aspect of emergency exercise scenario construction, scholars of various countries have done a lot of research [4]. In the book "The year 2000," the word "situation" was first put forward to enrich the content of "situation," especially the description of the present or future of the situation. In the application of scenarios, similarity measurement includes four steps as follows: locating 
repeated or nearly repeated scenarios, collecting scenario sets, identifying shared scenario points in scenarios, and determining the dependencies between scenarios [5]. Based on these four steps, a relatively effective method is semantic similarity analysis. As for the problem of locating scenes and identifying scene points, in the field of chemical accidents, some scholars believe that the failure to capture the loopholes in the identification scheme of common hazard sources leads to the situation that the atypical accident scene causes far more than the worst-case reference scene, so scene recognition technology is very important [6]. In view of multiple accidents in the field of traffic accidents, some scholars believe that the reason for not being able to give early warning is the inability to identify typical accident scenarios, so they put forward a new method of identifying typical scenarios [7]. Therefore, in foreign countries, scenarios are mostly applied in the early warning stage in the field of emergencies. In addition, some scholars have obtained some forward-looking early warning indicators that the current accident may develop into an "atypical" accident by comparing it with similar accidents that have happened.

In China, in the field of emergency management, scenarios were applied to preplan compilation [8], based on fuzzy rules reasoning, an emergency disposal scheme was quickly generated [9], an emergency strategy library building, etc., to try to solve the problem of plans and strategies in the library, but the strategy of the problem of the lack of a direct response to the scene information was extracted, which may also weaken the nature meaning of the "scene-to deal with" strategy, namely, the specific coping strategies. In order to solve this problem, some scholars put forward a solution based on the situation of a family tree design case where the longitudinal scene is divided into condition attribute and decision attribute, making every effort to achieve a one-to-one correspondence relation, but not for a single different emergency situation in the field of similarity calculation as well as the situation of a chain similarity algorithm to answer [10]. According to some scholars, elements are designed as a hazard-affected body, bearing the disaster of the trinity structure [11], and when connecting with the [12] multisource heterogeneous information fusion technology, knowledge yuan [13] situational inference technology, and methods to solve the problem, more is matched with the chain of events retrieval $[14,15]$, and based on scenarios, chain emergency similarity research is less.

In conclusion, in order to enable emergency management to achieve "scenario-response" mode, managers can better formulate emergency management measures. By comparing the structural similarity of the two events (one is to take effective control measures and the other is to prepare contingency plans for the absence of any contingency plans), we can make a reasonable decision on the emergency management measures that have not occurred. Based on the structural similarity, this paper proposes a similarity calculation method between the scene process of each event occurring in the emergency exercise scenario and the specific scenario, and then analyzes and predicts the process of the emergency exercise scenario to provide technical support for the construction of the emergency exercise. At the same time, safety accidents in oil and gas pipeline transportation and storage companies with similarities to the process of fluidized mining are analysed as case studies to provide theoretical references for the development of emergency response in the process of fluidized mining.

\section{Construction of an Emergency Exercise Scenario Structure Model and the Definition of Its Module}

This paper proposed a hierarchical structured emergency exercise scenario model (Figure 1), namely, the "EventEnvironment-State of scenario (SS)-Disposal of task (DT)Emergency action(EA)- Resources subject(RS)" model, hereinafter referred to as the EESTAR model. This model can fully reflect the structure of the emergency exercise scenario, the trend of the scenario's development during the exercise, and the measures and response subjects taken under the specific scenario state.

2.1. Event Module. In this paper, we defined the emergent event as a single emergency, referred to as an event which cannot be further subdivided according to its nature and mechanism. $E_{i}$ is the given event.

There are many event classification methods, and the type of subdivision is not uniform. The version content is also different. The level of events will affect the level of emergency exercise. The objects of the emergency exercise in this article are industrial production accidents, so we classify the events according to the level of the emergencies in the industrial accidents, from high to low, which are especially serious (I), major (II), larger (III), and general (IV), as shown in Table 1.

Assuming two events $E_{i}$ and $E_{j}$, the similarity between these two events is $S_{i j}^{\text {level }}$.

2.2. Environment Module. The environment has an important impact on the process of emergency exercise and emergency disposal. The scenarios under different environmental conditions have very different corresponding emergency exercises. The environment in this article includes six factors such as temperature $(\mathrm{T} / \mathrm{K})$, pressure $(\mathrm{P} /$ $\mathrm{kPa})$, relative humidity $(\varphi / \%)$, wind $(\mathrm{WD} / \mathrm{m} / \mathrm{s})$, day-night, and weather. Among them, day-night is divided into day, evening (dawn), and night, and the weather is divided into 4 cases, as shown in Table 2.

$E N_{i}$ is defined as the environment, supposed two events: $E_{i}$ and $E_{j}$, and their environmental similarity is $S_{i j}^{E N}$.

2.3. State of Scenario Model. The states of scenario refer to different states of emergencies in the time dimension. Over time, there will be an event development status chain, referred to as the status chain. Some of the states of scenarios include abnormal liquid levels, oil and gas leakage, oil spills, achieving explosive concentration, and combustion or explosion. The state chain represents the process of scenario 


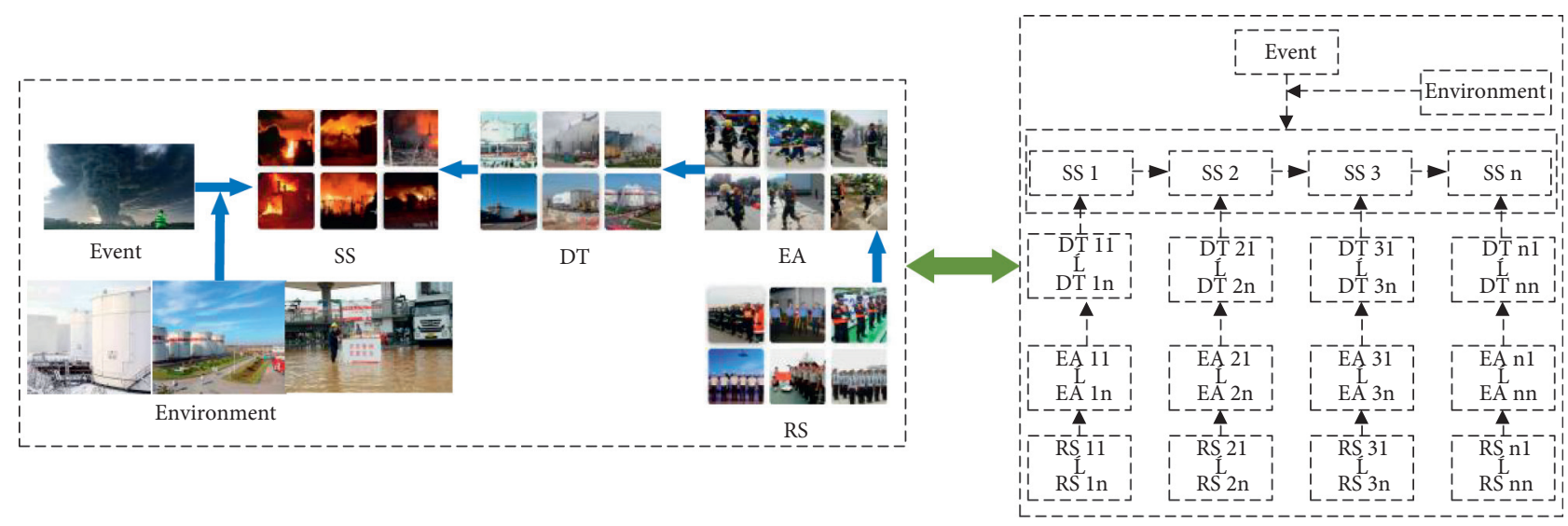

FIgURE 1: Structured emergency exercise scenario model.

TABLE 1: Criteria for dividing emergency levels.

\begin{tabular}{lccc}
\hline Level & Death & Severe injury & Direct economic loss \\
\hline Especially serious (I) & $\geq 30$ persons & $\geq 100$ persons & $\geq 10000$ million RMB \\
Major (II) & $10 \sim 30$ persons & $50 \sim 100$ persons & $5000 \sim 10000$ million RMB \\
Larger (III) & $3 \sim 10$ persons & $10 \sim 50$ persons & $1000 \sim 5000$ million RMB \\
General (IV) & $<3$ persons & $<10$ persons & $<1000$ million RMB \\
\hline
\end{tabular}

TABle 2: Classification of weather.

\begin{tabular}{lc}
\hline Weather conditions & Detailed classification \\
\hline Case 1: sunny, cloudy, dusk & None \\
Case 2: rainy & Light, moderate, heavy, \\
Case 3: snowy & rainstorm, sleet \\
Case 4: foggy & Light, moderate, heavy, blizzard \\
\hline
\end{tabular}

development, such as an abnormal liquid level-oil leakage-to achieve an explosive concentration-explosion. The nodes on the status chain are called status nodes, as shown in Figure 2.

The state of scenario is defined as $S A_{i}$, the states chain under $E_{i}$ is $C_{i}=\left\{S A_{i 1}, S A_{i 2}, \ldots, S A_{i n}\right\}$, and the number of states nodes is $m_{i}$. Assume events $A$ and $B$, their corresponding state name sets are $C_{i}$ and $C_{j}$, respectively. The state node similarity of these two events is defined as $S_{i j}^{m}$, and the state of scenario name similarity is defined as $S_{i j}^{S A}$.

2.4. Disposal of Task and Emergency Action Modules. Any different scenario on the state chain will correspond to the task, so the task is generated along with the generation of the scenario. A scenario will generate multiple tasks, forming a set of tasks. Each task is assigned to a different resource subject to complete, resulting in multiple emergency actions due to the functional differences of the different subjects. Therefore, in this article, there is a one-to-one correspondence between emergency actions and resource subjects, and we only count the similarity of emergency actions here. Measures to control the SS are DT, like abnormal liquid levels require a measure of control pressure or flow. An oil spill requires a measure of oil recovery. EA are the specific measures for DT, for example, to control pressure or flow, we need to take specific measures to locate leakage points, find leakage points, and accurately close valves.

The SS, DT, and EA are closely related and have a certain causal relationship due to their chronological order. Due to the difference in the state chain, the corresponding set of DT and EA will also differ. Therefore, in order to facilitate the calculation, we put all the tasks and emergency actions into the same set for unified. The vocabulary used in SS, DT, and EA needs to establish a unified standard database.

Defined DT as task $k_{i}$, EA as act ${ }_{i}$, the task set of $E_{i}$ as Task $_{i}$, Task $_{i}=\left\{\operatorname{task}_{i 1}, \operatorname{task}_{i 2}, \ldots, \operatorname{task}_{i n}\right\}$, and the set of $\operatorname{act}_{i}$ is Act $_{i}=\left\{\right.$ act $_{i 1}$, act $_{i 2}, \ldots$, act $\left._{i m}\right\}$. Assume that $E_{i}$ and $E_{j}$, their similarity of DT and similarity of EA are $S_{i j}^{\text {Task }}$ and $S_{i j}^{\text {Act }}$, respectively.

\section{Structured Similarity Calculation for Scenarios}

The structural characteristics of emergency exercise scenarios are as follows: a type of emergency that contains multiple emergencies; an emergency is composed of one or more scenarios to form a state chain; there are also similarities and differences between the state chains; corresponding to each state of the scenario, DT and EA have a one-to-many, or many-to-one relationship. Therefore, this section calculates and analyzes from the similarity of the states of the scenarios and similarity of the task actions.

3.1. The Similarity Calculation of the States of Scenarios. This step of the calculation is performed on the basis of similar events. The calculated similarity includes the level of the event, the environment, the state node, and the state chain. Finally, the four calculation results are combined 


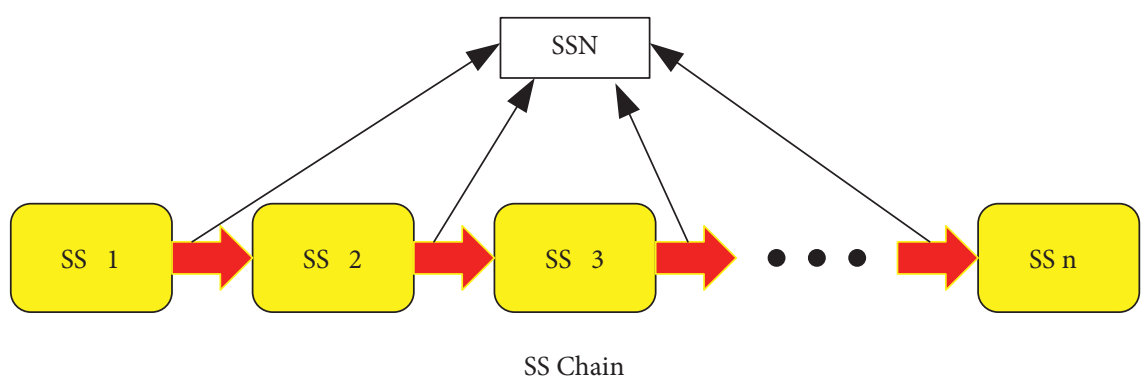

Figure 2: States chain and status nodes.

organically to obtain the similarity of the scenario state. The similarity calculation formula is as follows:

$$
A=1-\frac{|a-b|}{\max \{a, b\}} \text {. }
$$

3.1.1. The Similarity Calculation of the Level. The calculation formula of the level similarity is like formula (1). $S_{i j}^{\text {level }}$ is $A$, level $_{i}$ is $a$ level $_{j}$ is $b$, where level ${ }_{i}$ is the level of $E_{i}$, and the value is any one of $1-4$, which is based on Table 1 . $S_{i j}^{\text {level }} \in[0,1]$, when level ${ }_{i}$ and level ${ }_{j}$ are the same, the level of similarity between the two events reaches the highest.

3.1.2. The Similarity Calculation of the Environment. The environment includes 6 aspects, namely, temperature (T), pressure $(\mathrm{P})$, humidity $(\varphi)$, wind (WD), day and night (DN), and weather (WT). First, calculate the similarity of the first four.

The calculation formula of the similarity of the temperature is like formula (1). $S_{i j}^{T}$ is $\mathrm{A}, T_{i}$ is $a, T_{j}$ is $b$, where $T_{i}$ is the temperature of $E_{i}, T_{j}$ is the temperature of $E_{j}, \mathrm{~K}$ is the unit, and $S_{i j}^{T} \in[0,1]$. The closer the temperature of the two emergencies is, the higher $S_{i j}^{T}$ is.

The calculation formula of the similarity of the pressure is like formula (1). $S_{i j}^{P}$ is $A, P_{i}$ is $a, P_{j}$ is $b$, where $P_{i}$ is the pressure of $E_{i}, P_{j}$ is the pressure of $E_{j}, \mathrm{kp}$ is the unit, and $S_{i j}^{P} \in[0,1]$. The closer the pressure of the two emergencies is, the higher $S_{i j}^{P}$ is.

The calculation formula of the humidity is like formula (1). $S_{i j}^{\phi}$ is $A, \varphi_{i}$ is $a, \varphi_{j}$ is $b$, where $\varphi_{i}$ is the humidity of $E_{i}, \varphi_{j}$ is the humidity of $E_{j}, \%$ is the unit, and $S_{i j}^{\phi} \in[0,1]$. The closer the humidity of the two emergencies is, the higher $S_{i j}^{\phi}$ is.

The calculation formula of the similarity of the wind is like formula (1). $S_{i j}^{W D}$ is $A, W D_{i}$ is $a, W D_{j}$ is $b$. Where $W D_{i}$ is the wind of $E_{i}, W D_{j}$ is the wind of $E_{j}, \mathrm{~m} / \mathrm{s}$ is the unit, and $S_{i j}^{W D} \in[0,1]$. The closer the wind of the two emergencies is, the higher $S_{i j}^{W D}$.

The similarity of the day-night is $S_{i j}^{D N}$, and the comparison method is as follows:

(1) $S_{i j}^{D N}=1$ : all during day, night, or dusk (dawn)

(2) $S_{i j}^{D N}=0.5$ : one is at dusk (morning) and the other is during day or night

(3) $S_{i j}^{D N}=0$ : one is at day and the other is at night
The similarity of the weather is $S_{i j}^{W T}$. Referring to Table 1 , its assignment method is as follows:

(1) $S_{i j}^{W T}=1$ : for $E_{i}$ and $E_{j}$, in Table 1, the two columns are same

(2) $S_{i j}^{W T}=0.5$ : the first column is the same, and the second column is different

(3) $S_{i j}^{W T}=0$ : the first column is different

The similarity of the environment is $S_{i j}^{E N}$, which is as follows:

$$
S_{i j}^{E N}=\frac{S_{i j}^{T}+S_{i j}^{P}+S_{i j}^{\phi}+S_{i j}^{W D}+S_{i j}^{D N}+S_{i j}^{W T}}{6} .
$$

3.1.3. The Similarity Calculation of the State Node. The structural similarity of the state chain is measured by the difference in the number of nodes, which is convenient to distinguish the differences in the evolution of emergencies. The calculation formula of the similarity of the state node is like formula (1). $S_{i j}^{m}$ is A, $m_{i}$ is $a, m_{j}$ is $b$, where, $m_{i}$ is the number of nodes in the status chain $C_{i}, m_{j}$ is the number of nodes in the status chain $C_{j}$, and $S_{i j}^{m} \in[0,1]$. The closer the number of nodes connected by two chains is, the higher $S_{i j}^{m}$ is.

\subsubsection{The Similarity Calculation of the Name of the Scenario} State. This section calculates the state chain similarity based on a single state of scenario, as follows:

For $E_{i}$ and $E_{j}$, comparing $S A_{i n}$ and $S A_{j m}$, the formula for calculating the similarity of a single scenario state name is as follows:

$$
\operatorname{Sim}\left(S A_{i n}, S A_{j m}\right)=\frac{\text { same number of words }}{\text { maximum number of words }} .
$$

Compare $S A_{i n}$ with all the states in $E_{j}$, and take the maximum value to get $S_{i n}^{N a}$. $S_{i n}^{N a}$ is the similarity of $S A_{i n}$ in $E_{i}$. The calculation formula is as follows:

$$
\begin{aligned}
S_{i n}^{N a}= & \max \left[\operatorname{Sim}\left(S A_{i n}, S A_{j 1}\right), \operatorname{Sim}\left(S A_{i n}, S A_{j 2}\right), \ldots,\right. \\
& \left.\operatorname{Sim}\left(S A_{i n}, S A_{j m}\right)\right] .
\end{aligned}
$$

According to $S_{i n}^{N a}$, calculate $S_{i j}^{S A} . S_{i j}^{S A}$ is the similarity of the name of the state of scenario of $E_{i}$ and $E_{j}$. 


$$
\begin{aligned}
S_{i j}^{S A}= & {\left[\frac{\min \left(\sum_{x=1}^{n} S_{i x}^{N a} \text { or } \sum_{y=1}^{m} S_{j y}^{N a}\right)}{\max \left(\sum_{x=1}^{n} S_{i x}^{N a} \text { or } \sum_{y=1}^{m} S_{j y}^{N a}\right)} \times 0.7\right] } \\
& +\left(\frac{\sum_{x=1}^{n} S_{i x}^{N a}+\sum_{y=1}^{m} S_{j y}^{N a}}{n+m} \times 0.3\right) .
\end{aligned}
$$

3.1.5. The Similarity Calculation of the State of Scenario. Calculate the abovementioned 4 calculation results according to the corresponding weights to obtain the similarity of the state of scenario. The calculation formula is as follows:

$$
S_{i j}^{S S}=\left[\omega_{1}, \omega_{2}, \omega_{3}, \omega_{4}\right]\left[S_{i j}^{\text {level }}, S_{i j}^{E N}, S_{i j}^{m}, S_{i j}^{S A}\right]^{T},
$$

where, $\omega_{1}, \omega_{2}, \omega_{3}$, and $\omega_{4}$ are the weight values of $S_{i j}^{\text {level }}, S_{i j}^{E N}, S_{i j}^{m}$, and $S_{i j}^{S A}$, respectively.

3.2. The Similarity Calculation of the Task and Action. The calculation of this part includes the similarity of DT and the similarity of EA. Finally, the two results are combined to obtain the final result. And these two calculation methods are the same as part 4 in Section 3.1.

$$
\begin{aligned}
\operatorname{Task}_{i} & =\left\{\operatorname{task}_{i 1}, \operatorname{task}_{i 2}, \ldots, \operatorname{task}_{i n}\right\}, \\
\operatorname{Act}_{i n} & =\left\{\operatorname{act}_{i 1}, \operatorname{act}_{i 2}, \ldots, \operatorname{act}_{i m}\right\},
\end{aligned}
$$

$S_{i j}^{\text {Task }}$ is the similarity of DT,

$$
\begin{aligned}
S_{i j}^{\text {Task }}= & {\left[\frac{\min \left(\sum_{x=1}^{n} \operatorname{Task}_{i x}^{N a} \text { or } \sum_{y=1}^{m} \operatorname{Task}_{j y}^{N a}\right)}{\max \left(\sum_{x=1}^{n} \operatorname{Task}_{i x}^{N a} \text { or } \sum_{y=1}^{m} \operatorname{Task}_{j y}^{N a}\right)} \times 0.7\right] } \\
& +\left(\frac{\sum_{x=1}^{n} \operatorname{Task}_{i x}^{N a}+\sum_{y=1}^{m} \operatorname{Task}_{j y}^{N a}}{n+m} \times 0.3\right)
\end{aligned}
$$

$S_{i j}^{\text {Act }}$ is the similarity of EA,

$$
\begin{aligned}
S_{i j}^{\mathrm{Act}}= & {\left[\frac{\min \left(\sum_{x=1}^{n} \mathrm{Act}_{i x}^{N a} \text { or } \sum_{y=1}^{m} \mathrm{Act}_{j y}^{N a}\right)}{\max \left(\sum_{x=1}^{n} \operatorname{Act}_{i x}^{N a} \text { or } \sum_{y=1}^{m} \mathrm{Act}_{j y}^{N a}\right)} \times 0.7\right] } \\
& +\left(\frac{\sum_{x=1}^{n} \mathrm{Act}_{i x}^{N a}+\sum_{y=1}^{m} \mathrm{Act}_{j y}^{N a}}{n+m} \times 0.3\right),
\end{aligned}
$$

where, both $S_{i j}^{\text {Task }}$ and $S_{i j}^{\text {Act }}$ are between $0-1$.

Above all, the similarity of the task and action is $S_{i j}^{T A}$,

$$
S_{i j}^{T A}=\left[\omega_{1}, \omega_{2}\right]\left[S_{i j}^{\text {Task }}, S_{i j}^{\text {Act }}\right]^{T}
$$

where, $\omega_{1}$ and $\omega_{2}$ are the weight values of $S_{i j}^{\text {Task }}$ and $S_{i j}^{\text {Act }}$, respectively.

\subsection{The Similarity of the Scenario and Similarity Evaluation.} The similarity of the scenario in the emergency exercise is a combination of the abovementioned similarity, which is calculated as follows:

$$
S_{i j}=\left[\omega_{1}, \omega_{1}\right]\left[S_{i j}^{S S}, S_{i j}^{T A}\right]^{T}
$$

where, $\omega_{1}$ and $\omega_{2}$ are the weight values of $S_{i j}^{S S}$ and $S_{i j}^{T A}$, respectively.

Divided $S_{i j}$ into five levels, which are dissimilarity, low similarity, medium similarity, high similarity, and extremely high similarity. Then, the specific similarity evaluation is carried out according to the classification of corresponding emergencies, as shown in Table 3.

\section{The Example Analysis}

According to the "5.13" deflagration accident at Alashan pass in the Xinjiang Uygur autonomous region and the oil spill accident at Guazhou oil transfer station in Gansu province provided by the CNPC Western Pipeline Company, relevant information of emergency exercise scenarios in these two accidents is presented. According to the relevant terms in the emergency case of oil and gas pipelines, the " 5.13 " deflagration accident at Alashan pass and the oil spill accident at Guazhou oil transfer station were built, as shown in Figures 3 and 4 .

Step 1. Calculate the state of scenario's structural similarity

Both incidents are classified as general emergencies in terms of oil spills, and both are classified as class IV incidents. For the "5.13" deflagration accident at Alashan pass, the local temperature is $295 \mathrm{~K}$, the air pressure is $945 \mathrm{KP}$, the humidity is $30 \%$, the wind speed is $8.7 \mathrm{~m} / \mathrm{s}$, and the weather is sunny during the day. For the oil spill accident at Guazhou oil transfer station, the local temperature is $287 \mathrm{~K}$, the air pressure is 925 $\mathrm{KP}$, the humidity is $34 \%$, the wind speed is $3.5 \mathrm{~m} / \mathrm{s}$, and the weather is cloudy at dusk. There are three cases of situational chain nodes in two accidents. So, according to formulas (1) and (2), the following results are obtained:

$$
\begin{aligned}
S_{i j}^{\text {level }} & =1-\frac{|4-4|}{\max \{4,4\}}=1, \\
S_{i j}^{E N} & =\frac{0.97+0.98+0.88+0.40+0.5+1}{6}=0.79, \\
S_{i j}^{m} & =1-\frac{|3-3|}{\max \{3,3\}}=1 .
\end{aligned}
$$

To calculate the state of the scenario name's similarity, the contents of $C_{i}$ and $C_{j}$ of the accident state of the scenario are first clarified.

$C_{i}=\{$ abnormal liquid level of a waste oil tank, spillover of leaking waste oil, crude oil seam meets the requirements of combustion and explosion\}

$C_{j}=\{$ inspector finds abnormal conditions, determines information of leaking crude oil, and diffuses leaking crude oil\}

$S A_{i 1}=$ abnormal liquid level of a waste oil tank

$S A_{i 2}=$ spillover of leaking waste oil

$S A_{i 3}=$ crude oil seam meets the requirements of combustion and explosion 
TABLE 3: Evaluation matrix similarity of emergency exercise scenarios.

\begin{tabular}{|l|c|c|c|c|c|}
\hline$S_{i j}$ & $<60 \%$ & $60 \% \sim 70 \%$ & $70 \% \sim 80 \%$ & $80 \% \sim 90 \%$ & $\geq 90 \%$ \\
\hline Similarity & Dissimilarity & Low similarity & Medium similarity & High similarity & Extremely high similarity \\
\hline Level I & reject & reject & reject & reject & accept \\
\hline Level II & reject & reject & reject & accept & accept \\
\hline Level III & reject & reject & accept & accept & accept \\
\hline Level IV & reject & accept & accept & accept & accept \\
\hline
\end{tabular}

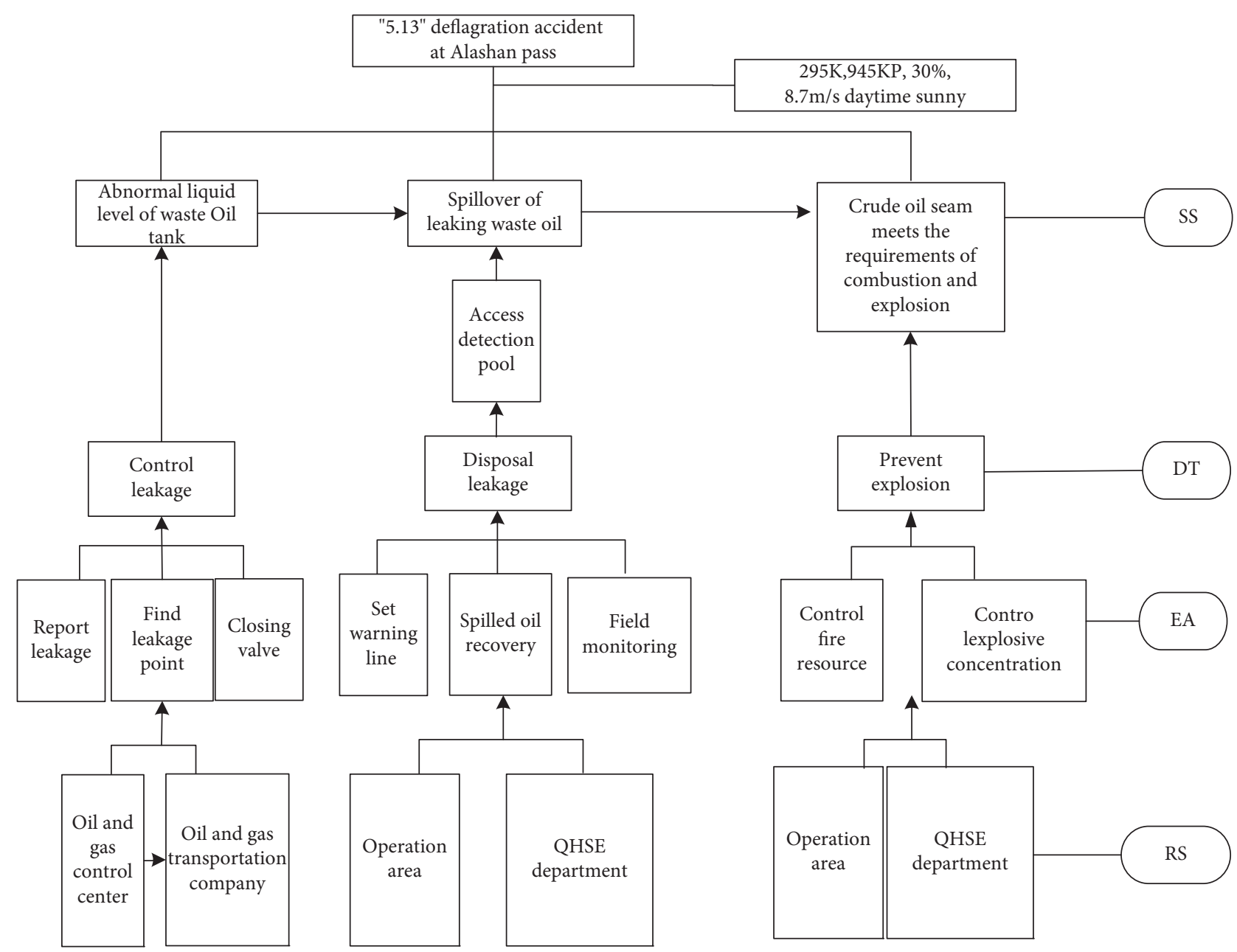

Figure 3: Scenarios of the "5.13" deflagration accident at Alashan pass.

$S A_{j 1}=$ inspector finds abnormal conditions

$S A_{j 2}=$ determines information of leaking crude oil

$S A_{j 3}=$ diffusion of leaking crude oil

By pairwise comparison between any node in $S A_{\text {in }}$ and $S A_{j m}$, the following results can be obtained according to formulas (1) and (2):

$$
\operatorname{Sim}\left(S A_{i 1}, S A_{j 1}\right)=1 / 7=0.14
$$

$\operatorname{Sim}\left(S A_{i 1}, S A_{j 2}\right)=1 / 7=0.14$

$\operatorname{Sim}\left(S A_{i 1}, S A_{j 3}\right)=1 / 7=0.14$

$\operatorname{Sim}\left(S A_{i 2}, S A_{j 1}\right)=0 / 5=0$

$\operatorname{Sim}\left(S A_{i 2}, S A_{j 2}\right)=2 / 6=0.33$

$\operatorname{Sim}\left(S A_{i 2}, S A_{j 3}\right)=2 / 5=0.40$

$\operatorname{Sim}\left(S A_{i 3}, S A_{j 1}\right)=0 / 10=0$

$\operatorname{Sim}\left(S A_{i 3}, S A_{j 2}\right)=2 / 10=0.20$ 


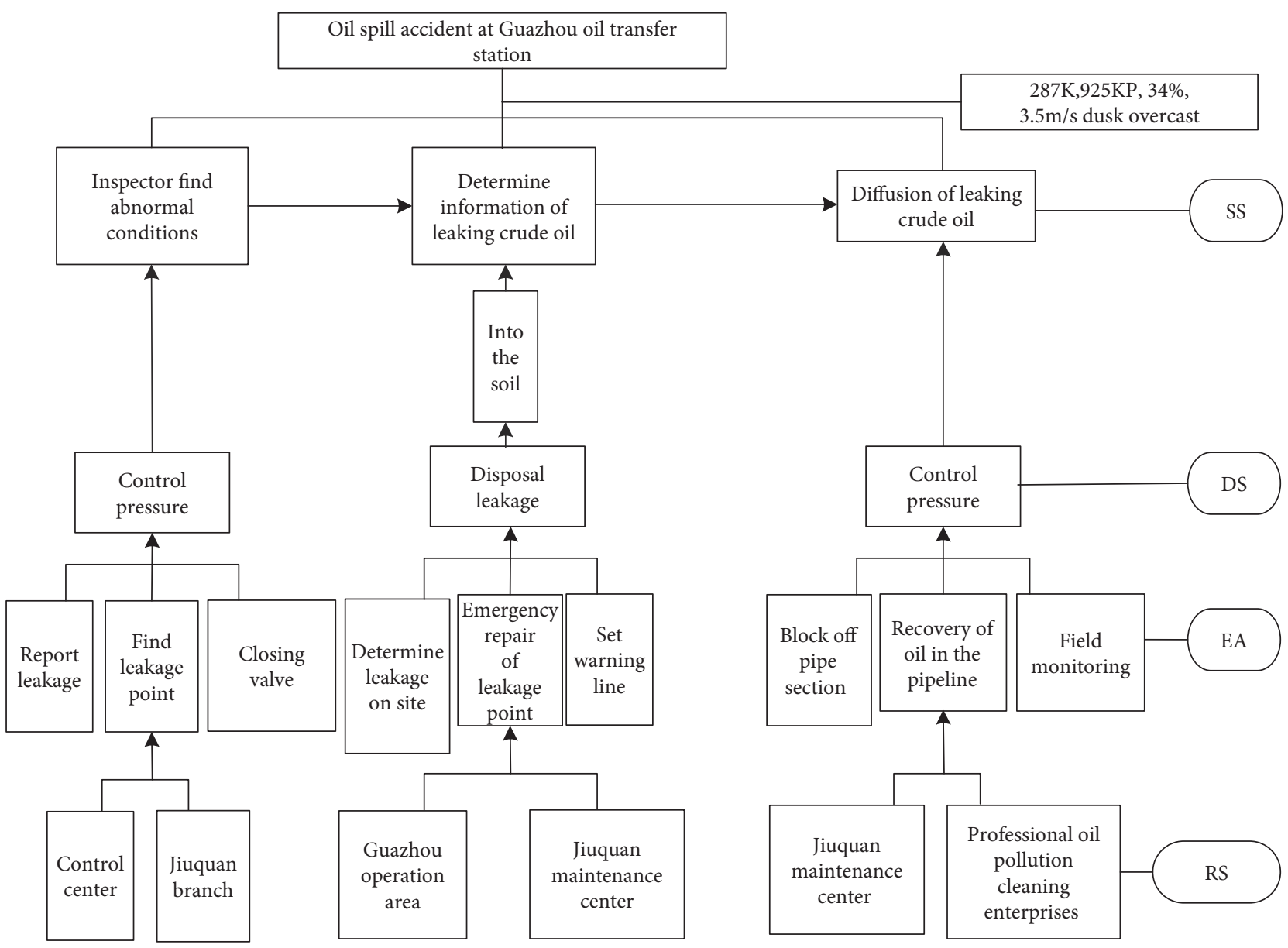

FIgURE 4: Scenarios of the oil spill accident at Guazhou oil transfer station.

$\operatorname{Sim}\left(S A_{i 3}, S A_{j 3}\right)=2 / 10=0.20$

According to formula (4), $S A_{i 1}$ 's similarity is $0.14, S A_{i 2}$ 's similarity is $0.40, S A_{i 3}$ 's similarity is $0.20, S A_{j 1}$ 's similarity is $0.14, S A_{j 2}$ 's similarity is 0.33 , and $S A_{j 3}$ 's similarity is 0.40 . Through formula (5), the state of the scenario name's similarity is obtained.

$$
\begin{aligned}
S_{i j}^{S A}= & \left(\frac{0.14+0.40+0.2}{0.14+0.33+0.40} \times 0.7\right) \\
& +\left(\frac{0.14+0.40+0.2+0.14+0.33+0.40}{6} \times 0.3\right)
\end{aligned}
$$

$=0.68$.

Assuming that the decision-maker assigns $\omega_{1}, \omega_{2}, \omega_{3}$, and $\omega_{4}$ as $0.3,0.2,0.2$, and 0.3 , respectively, according to the needs of emergency handling in the real situation. Through formula (6), the similarity of the two emergency state of scenario is as follows:

$$
S_{i j}^{S S}=[0.3,0.2,0.2,0.3][1,0.78,1,0.68]^{T}=0.814
$$

\section{Step 2. Calculate the task and act's similarity}

The calculation methods of task similarity and action similarity in the task and action similarity are the same as the calculation methods of state of scenario name's similarity, so this paper does not introduce the calculation details of task similarity and action similarity. According to formulas (8) and (9), task similarity and action similarity are obtained.

$$
\begin{aligned}
S_{i j}^{\text {Task }} & =0.9, \\
S_{i j}^{\text {Act }} & =0.81 .
\end{aligned}
$$

Assuming that the decision-maker assigns 0.3 and 0.7 to $\omega_{1}$ and $\omega_{2}$ according to the actual situation of emergency action and disposal tasks in the emergency exercise scenario, then the task and action similarity can be calculated according to formula (10).

$$
S_{i j}^{T A}=[0.3,0.7][0.9,0.81]^{T}=0.837 .
$$

Step 3. Evaluation of emergency exercise scenario similarity

Based on the state of scenario similarity and task and action similarity obtained in Steps 1 and 2, the decisionmaker assigned $\omega_{1}$ and $\omega_{2}$ value of 0.4 and 0.6 , respectively, and the scenario's similarity was calculated by formula (11).

$$
S_{i j}=[0.4,0.6][0.814,0.837]^{T}=0.8278 .
$$

According to Table 3, the emergency exercise's scenarios of the " 5.13 " deflagration accident in Alashan pass and the 
TABLE 4: Index values of emergency drills scenario similarity.

\begin{tabular}{lccccccccc}
\hline Similarity index & $S_{i j}^{\text {level }}$ & $S_{i j}^{E N}$ & $S_{i j}^{m}$ & $S_{i j}^{S A}$ & $S_{i j}^{S S}$ & $S_{i j}^{\text {Task }}$ & $S_{i j}^{\text {Act }}$ & $S_{i j}^{T A}$ & $S_{i j}$ \\
\hline Value & 1 & 0.79 & 1 & 0.68 & 0.8144 & 0.9 & 0.81 & 0.837 & 0.8278 \\
\hline
\end{tabular}

oil spill accident at Guazhou oil transfer station have a high similarity. Since these two events are level IV, the similarity of their emergency exercise scenario is considered acceptable. The index values calculated for emergency drill scenario similarity are shown in Table 4.

\section{Conclusion}

The emergency exercise scenario structured model of "Event-Environment-State of scenario-Disposal of taskEmergency action- Resources subject" proposed in this paper studies the whole process of emergency response from the perspective of a scenario, constitutes a sequential chain of scenario, task and action, and improves the auxiliary ability for emergency decision-making. Based on this model, the state scenario chain of emergencies is constructed, and the correlation similarity is obtained by comparing the structure name similarity of the state scenario chain. By comparing the similarity between the disposal task and emergency action, the corresponding similarity is obtained. Through such a calculation, not only the suggested fluidized mining emergency plan of the event in the current situation can be obtained, but also the disposal task and emergency action of the corresponding situation in the predicted development trend can be suggested.

(1) Structural model of emergency exercise's scenario. The emergency exercise's scenario-structured model of "event-environment-state of scenario-disposal task-emergency action-resource subject” is proposed in this paper.

(2) Emergency exercise's scenario similarity calculation. By using the structure, content, and name of the state of the scenario chain to obtain correlation similarity. Through the task and action on the state of the scenario status chain, obtain the corresponding similarity. Finally, the scenario similarity is obtained.

(3) Similarity evaluation results. Obtain a suggested emergency plan for events in the current situation. Predict the disposal tasks and emergency actions of the corresponding scenarios under the development trend.

(4) This paper mainly provides a way to provide emergency management. The standard database of vocabulary, weight parameters, and evaluation matrix for the fluidized mining similarity needs to be optimized in practice.

\section{Data Availability}

The data used to support the findings of this study are available from the corresponding author upon request.

\section{Conflicts of Interest}

The authors declare that they have no conflicts of interest.

\section{Acknowledgments}

The authors thank the support of the CNPC West Pipeline Company. This research was funded by the National Key Research and Development Program of China (Grant number: 2016YFC0801800) and the Beijing Natural Science Foundation (Grant number: 2174084). Financial support was provided by the National Basic Research Program of China (Grant numbers: 51774291 and 51864045).

\section{References}

[1] A. A. A. Ahmed and M. M. Dey, "Accounting disclosure scenario: an empirical study of the banking sector of Bangladesh," Accounting \& Management Information Systems, vol. 19, p. 2, 2011.

[2] N. Paltrinieri, J. Wilday, M. Wardman, and V. Cozzani, "Surface installations intended for carbon capture and sequestration: atypical accident scenarios and their identification," Process Safety and Environmental Protection, vol. 92, no. 1, pp. 93-107, 2014.

[3] N. Paltrinieri, D. Nicolas, S. Ernesto, W. Mike, and C. Valerio, "Towards a new approach for the identification of atypical accident scenarios," Journal of Risk Research, vol. 16, pp. 337-354, 2013.

[4] N. Paltrinieri, K. Øien, and V. Cozzani, "Assessment and comparison of two early warning indicator methods in the perspective of prevention of atypical accident scenarios," Reliability Engineering \& System Safety, vol. 108, pp. 21-31, 2012.

[5] N. Paltrinieri, F. Khan, and V. Cozzani, "Coupling of advanced techniques for dynamic risk management," Journal of Risk Research, vol. 18, no. 7, pp. 910-930, 2015.

[6] M. M. Sood, A. R. Sood, and R. Richardson, "Emergency management and commonly encountered outpatient scenarios in patients with hyperkalemia," Mayo Clinic Proceedings, vol. 82, no. 12, pp. 1553-1561, 2007.

[7] K. B. Athreya and P. Ney, "Atypical scenarios identification by the DyPASI procedure: application to LNG," Chemical Engineering Transactions, vol. 24, pp. 1171-1176, 2011.

[8] L. Zappini, S. Marchesi, A. Polo, F. Viani, and A. Massa, "Evolutionary optimization strategies applied to wireless fleet management in emergency scenarios," in Proceedings of the 2015 IEEE 15th Mediterranean Microwave Symposium (MMS), Lecce, Italy, November 2015.

[9] R. Torres, L. Mengual, O. Marban, S. Eibe, E. Menasalvas, and B. Maza, "A management Ad Hoc networks model for rescue and emergency scenarios," Expert Systems with Applications, vol. 39, no. 10, pp. 9554-9563, 2012.

[10] L. Lanteri, B. Daniele, M. Morelli, F. Dutto, D. Giordan, and A. Manconi, "Rockfall analysis during emergency scenarios," in Proceedings of the Engineering Geology for Society and Territory-Volume 5, Springer International Publishing, Torino, Italy, September 2014. 
[11] D. Alexander, "Scenario methodology for teaching principles of emergency management," Disaster Prevention and Management: International Journal, vol. 9, no. 2, pp. 89-97, 2000.

[12] Y. Xiaofang and L. Hongxia, "Notice of retraction research on the IDSS of unconventional emergency management based on scenario analysis and CBR," in Proceedings of the IEEE International Conference on Emergency Management \& Management Sciences, IEEE, August 2011.

[13] T. Robinson, T. M. Wilson, T. R. Davies, C. Orchiston, and J. Thompson, "Using the co-production of knowledge for developing realistic natural disaster scenarios for small-tomedium scale emergency management exercises," in Proceedings of the Agu Fall Meeting, San Francisco, CA, USA, December 2014.

[14] N. Dimakis, A. Filippoupolitis, and E. Gelenbe, "Distributed building evacuation simulator for smart emergency management," The Computer Journal, vol. 53, no. 9, pp. 1384-1400, 2010.

[15] G. Eleftherakis, "Stigmergy inspired approach to enable agent communication in emergency scenarios," in Proceedings of the Balkan Conference on Informatics Conference, Craiova, Romania, September 2015. 